\title{
A Longitudinal Study of Physical Activity to Improve Sleep Quality During Pregnancy
}

This article was published in the following Dove Press journal:

Nature and Science of Sleep

\author{
Liwei Tan ${ }^{1, *}$ \\ Jiaojiao Zou' ${ }^{1, *}$ \\ Yunhui Zhang ${ }^{2}$ \\ Qing Yang ${ }^{3}$ \\ Huijing Shi ${ }^{1}$
}

'Department of Maternal, Child and Adolescent Health, School of Public Health, Fudan University, Shanghai, People's Republic of China; ${ }^{2}$ Department of Environmental Health, School of Public Health, Fudan University, Shanghai, People's Republic of China; ${ }^{3}$ Department of Child Care, The Maternal and Child Healthcare Institute of Songjiang District, Shanghai, People's Republic of China

*These authors contributed equally to this work
Correspondence: Huijing Shi; Qing Yang Tel +86-2I-54237022; +86 02I-3762I588 Fax +862I-54237022

Email hjshi@fudan.edu.cn; yqzjj888@।26.com
Purpose: To explore the association between maternal physical activity (PA) and sleep quality during pregnancy, and the necessary PA level at different gestational stages to attain improved sleep quality.

Methods: A total of 2443 participants were recruited from the Shanghai Maternal-Child Pairs Cohort (Shanghai MCPC) study, who had completed questionnaires including the Pittsburgh Sleep Quality Index (PSQI) and the International Physical Activity Questionnaire (IPAQ) at gestational weeks (GW) of 12-16 and 32-36. PSQI scores and their seven components at the two GW were compared, as were PSQI scores at 12-16 and 32-36 GW and the increment in PSQI relative to PA. Regression analysis was conducted to assess the effect of PA and its change on the total PSQI score at different GW.

Results: The mean PSQI scores increased significantly during pregnancy, from $6.30 \pm 3.01$ at $12-16 \mathrm{GW}$ to $7.23 \pm 3.47$ at 32-36 GW. Compared with women in low PA level, moderate levels of PA at both $12-16 \mathrm{GW}$ and $32-36 \mathrm{GW}$ were significantly reduced PSQI scores of 0.42 (95\% CI:-0.68,-0.16) and 0.32 (95\% CI:-0.63,-0.01), respectively. At 32-36 GW, high PA level also significantly decreased PSQI score, with a greater decline than moderate PA level. (AOR $=-0.87,95 \% \mathrm{CI}:-1.57,-0.18)$. PA increment from $12-16$ to $32-36$ weeks of pregnancy created a significant decline of 0.54 in PSQI scores.

Conclusion: The study revealed sleep quality was worse at the third trimester and moderate PA level had the potential for improvement of sleep quality both in the first and the third trimester. High PA level was also beneficial to improve sleep quality of pregnant women in the third trimester.

Keywords: gestational weeks, pregnancy, sleep quality, physical activity

\section{Introduction}

Good sleep quality is essential for women at all stages of pregnancy, and improving sleep quantity and quality during overall gestation is of utmost importance for positive obstetric outcomes and an optimal health-related quality of life. For example, several studies have shown that poor sleep quality increased the risk for preterm birth and cesarean section $^{1,2}$ and enhanced the likelihood of 3.34-fold for postpartum depression. $^{3-7}$ Regarding fetal outcomes, a meta-analysis ${ }^{8}$ synthesized the literature related to maternal sleep and poor fetal outcomes and demonstrated that poor sleep quality or sleep syndrome may manifest disadvantages of altered growth, gestational length, and even fetal death. Sleep during pregnancy is dynamic, and its quality is typically worse than that in the general population. ${ }^{9}$ With an increase of gestational weeks, sleep quality during pregnancy is also affected by factors that are not easy to control, including social, mental, and physical factors, besides the alterations in 
hormones and physiology. ${ }^{10,11}$ Gestational weeks and prenatal emotional problems, such as depression, anxiety, and stress, have been emphasized in previous studies, and these factors showed associations with sleep quality among pregnant women. ${ }^{12-15}$ In addition, the results of a randomized controlled trial ${ }^{16}$ showed that moderate-intensity water exercise at 20-37 gestational weeks would improve the quality of sleep in pregnant women, both subjectively and objectively in terms of latency, duration and efficiency.

However, there is a paucity of research that explores the association between maternal physical activity and sleep quality during pregnancy, or the proper level of physical activity at different gestational weeks to attain better sleep quality. A large amount of literature supports the concept that physical activity can improve sleep quality in the non-pregnant population, of which some studies report that exercise improves sleep quality and continuity. $^{17-19}$ A systematic review ${ }^{20}$ in 2018 claimed that exercise improved sleep quality among patients with primary and secondary insomnia. A cross-sectional study of university students found that poor sleep quality was associated with insufficient physical activity, and a 10-year follow-up study provided corroboration that women who maintained higher levels or increased their levels of leisure-time physical activity were protected from selfreported insomnia. ${ }^{21}$ There are, however, several other cross-sectional studies that investigated the association between physical activity and sleep quality during pregnancy, and these showed equivocal results. Baker et al found that exercise during early pregnancy was correlated with greater sleep continuity. ${ }^{22}$ However, Soner Ölmez ${ }^{23}$ found no significant difference of sleep quality between groups of pregnant women with different levels of exercise, who are between 5 and 40 gestational weeks. In recent years, people have generally come to realize the importance of physical activity during pregnancy. The Physical Activity Guidelines for Americans and 2019 Canadian Guideline for Physical Activity Throughout Pregnancy ${ }^{24,25}$ recommended that pregnant women perform at least 150 minutes ( 2 hours and 30 minutes) of moderate-intensity aerobic activity per week during pregnancy. The former guidelines also suggested that pregnant women who habitually engaged in vigorous-intensity aerobic activity or who were physically active before pregnancy can continue these activities during pregnancy; however, the guidelines did not provide detailed suggestions for different trimesters, and because of differences in physical and psychologic factors, the requirements for physical activity are probably different.

Prospective studies will be critical to evaluate the potential impacts of physical activity on sleep quality. In the present study, we observed maternal sleep at 12-16 gestational weeks and at 32-36 gestational weeks, respectively. In addition, we examined the prospective association between physical activity and subjective sleep quality in pregnant women. Physical activities were measured twice using the International Physical Activity Questionnaire (IPAQ short version), and we measured indices of subjective sleep quality as well using the Pittsburgh Sleep Quality Index (PSQI). We also explored the effect of changes in physical activity on maternal sleep quality. As few researchers have examined the relationship between physical activity and sleep quality during pregnancy, we hypothesized that physical activity would vary during pregnancy and necessary physical activity levels at different gestational stages might play a role in clinical guidance, in order to improve the quality of sleep during pregnancy.

\section{Materials and Methods Design and Sample}

Population samples of the present study were from the Shanghai Maternal-Child Pairs Cohort (Shanghai MCPC), which was funded by two programs in Shanghai. We recruited pregnant women at 12-16 gestational weeks from two regional maternity hospitals of Shanghai's Pudong and Songjiang Districts hospitals from April 2016. We finally analyzed 2443 participants who had completed questionnaires that included the Pittsburgh Sleep Quality Index (PSQI) and the International Physical Activity Questionnaire (IPAQ) at gestational weeks (GW) 12-16 and 32-36. The inclusion criteria were pregnant women $\geq 20$ years of age, living in Shanghai currently, being able to read Chinese, and willing to participate in the cohort study. Pregnant women with syphilis or HIV were excluded. All of the participants included were singleton pregnancy. Sociodemographic information (eg, birth date, employment, and education level) was collected via self-report questionnaires, and medical records were obtained from the hospital database. This cohort study was reviewed and approved by the Ethics Committee of the School of Public Health, Fudan University. All participants provided written informed consent, and this study was conducted in accordance with the Declaration of Helsinki. 


\section{Instruments}

\section{Sleep Quality: Pittsburgh Sleep Quality Index}

The Pittsburgh Sleep Quality Index (PSQI) is a self-rating questionnaire that assesses sleep quality and disturbances over a 1-month interval of time. PSQI total scores showed good internal consistency and construct validity. Cronbach's alpha for the PSQI (items were the 7 component subscale scores) was 0.76 , goodness-of-fit index (GFI) and comparative fit index (CFI) were 0.95 and 0.91 , respectively, RMSEA was 0.13 and $\chi 2$ (minimum discrepancy) divided by degrees of freedom (CMin/DF) was 2.6. ${ }^{26}$ Nineteen individual items generated seven "component" scores: subjective sleep quality, sleep latency, sleep duration, habitual sleep efficiency, sleep disturbances, use of sleeping medication, and daytime dysfunction. ${ }^{27}$ Each of the sleep components yields a score ranging from 0 to 3 , with 3 indicating the greatest dysfunction. The sleep component scores are summed to yield a total score ranging from 0 to 21 , with the higher total score (referred to as global score) indicating worse sleep quality. A person was defined as a poor sleeper if her/his global score was $>5$. The PSQI is widely used to evaluate the sleep quality of various populations in different countries. Sleep quality was investigated at 12-16 gestational weeks (early pregnancy) and at 32-36 gestational weeks (later pregnancy). $\triangle \mathrm{PSQI}$ was defined as the score increment (or decrement) from 12-16 gestational weeks to 32-36 gestational weeks.

\section{Physical Activity Level: International Physical Activity Questionnaire (IPAQ Short Version)}

The International Physical Activity Questionnaire (IPAQ short version $)^{28}$ is frequently used to assess physical activity (PA) level in the general adult population including pregnant women and has good reliability and validity in pregnant women, with the test-retest intraclass-correlation of different PA level ranging $0.81-0.84 .{ }^{29-31}$ There were 7 questions in the questionnaire, and 6 of them referred to the intensity of personal PA, including the frequency of walking, running, or playing sports. We investigated PA twice (at 12-16 gestational weeks and at 32-36 gestational weeks). MET (Metabolic Equivalents, MET) $^{28}$ is an important indicator that reflects the body's metabolic level and energy expenditure. Based on the metabolic equivalent table, the average metabolic equivalent value of physical activity was assigned per hour. According to corresponding standards, ${ }^{32,33} \mathrm{PA}$ levels were divided into three categories: low, moderate, and high. Met levels were defined as walking $=3.3$ Mets, moderate intensity $=4.0$ Mets, and vigorous intensity $=8.0$ Mets. Total MET-minutes $/$ week $=$ Walk $($ METs*min*days $)+$
Mod (METs*min*days) + Vig (METs*min*days). The high category was considered to include those individuals who participated in vigorous-intensity activity at least 3 days and accumulated at least 1500 MET-minutes/week, or 7 or more days of any combination of walking, and moderate- or vigorous-intensity activities that accumulated at least 3000 MET-minutes/week. We defined the moderate category to be equivalent to 3 or more days of vigorous activity for at least 20 minutes per day, or 5 or more days of moderateintensity activity and/or walking of at least 30 minutes per day, or 5 or more days of any combination of walking, moderate-intensity, or vigorous-intensity activities that achieved a minimum of at least 600 MET-minutes/week. The low category was defined to include those individuals who could meet any criteria for the two aforementioned categories. PA was also investigated at 12-16 gestational weeks and at $32-36$ gestational weeks separately. The change from 12-16 to 32-36 gestational weeks was defined as the $\triangle \mathrm{PA}$ (MET-minutes/week difference). Groups were divided by increasing or not increasing PA, which consisted of the increased group and the non-increased group.

\section{Sociodemographic Factors}

Age of pregnant women: The difference between the date of the survey and the date of birth of the pregnant women was calculated and divided into three categorical groups: 20-25 years old, 26-30 years old, and 31 years old and above. The educational level was divided into high school and below, junior college, and university and above. The annual income of the core family was obtained by asking "the total income of your core family last year (including various sources such as wages and stocks)" and it was translated into low, middle, and high-income groups. We also asked the pregnant women in the survey whether they worked or not in the early or middle pregnancy, and we divided them into two groups.

\section{Potential Confounders Pre-Pregnancy BMI}

Height and pre-pregnancy weight were based on self-reported data from the pregnant women's baseline interview. Height was measured in $\mathrm{m}$, with accuracy to two decimal places, and weight was in $\mathrm{kg}$, with accuracy measured to one decimal place. Body mass index (BMI) was calculated by dividing body weight $(\mathrm{kg})$ by height-squared $(\mathrm{m} 2)$, with the results determined to one decimal place. Pre-pregnant women were categorized as "underweight" (BMI $<18.5 \mathrm{~kg} / \mathrm{m} 2$ ), "normal 
weight" $(18.5 \leq \mathrm{BMI}<24 \mathrm{~kg} / \mathrm{m} 2)$, and "overweight or obese" $(\mathrm{BMI} \geq 24 \mathrm{~kg} / \mathrm{m} 2) .{ }^{34}$

\section{Underlying Diseases}

We asked pregnant women whether they had been diagnosed with hypertension, diabetes, asthma, pneumonia, tuberculosis, periodontitis, hyperthyroidism, hypothyroidism, hepatitis, congenital heart disease, nephritis, anemia, endometriosis before becoming pregnant, uterine fibroids, or polycystic ovary syndrome; 1 or more of which was defined as having a history of underlying disease.

\section{Parity and History of Abortion}

Parity was determined by calculating the total number of live births delivered in the past and with gestational weeks $\geq 28$ weeks divided into birth history or no birth history. Abortion history was designated as the number of abortions and medical abortions, divided into a history of abortion or no history of abortion.

\section{Psychological Stress: Life-Events Scale for Pregnant Women}

We measured maternal stress during pregnancy using the life events scale for pregnant women (LESPW). The LESPW has demonstrated good reliability with a Cronbach's alpha of 0.9607 for total and $0.7756,0.8796,0.8924$ and 0.9024 for four subscales, respectively. ${ }^{35,36}$ The scale contains 53 life events, including family life, work and study, social relations, and other aspects during pregnancy. Based on the actual events that occurred, the pregnant women then selected an answer, and the weighted score was calculated. A total score higher than the critical point of 375 points showed that the events were comparatively more stressful during pregnancy. ${ }^{35}$

\section{Statistical Analyses}

Data were statistically analyzed by SPSS (version 22.0). PA levels as categorical variable were the primary exposure variable in all analyses. Descriptive statistics were used to characterize our population sample. Covariates were sociodemographic factors (eg, age, education, working status), medical records (eg, pre-pregnant BMI, parity, history of abortion), and psychological stress. PSQI scores and their seven components during the periods $12-16$ and 32-36 gestational weeks were compared by paired-sample $t$-tests and the correlation analysis was conducted between PSQI scores at the two pregnancy stages. PSQI scores for 12-16 gestational weeks and 32-36 gestational weeks and the increment in PSQI were compared among the different levels of physical activity, as well as the groups of potentially related factors (such as sociodemographic factors, medical records, and psychologic stress) by Bonferroni tests and Student's $t$-tests or one-way ANOVAs. We conducted multiple logistic regression analyses to evaluate the effect of PA and its changes on the total PSQI score at different stages of gestation while also controlling potential covariates (such as sociodemographic factors, medical records and psychological stress, etc.). We selected these confounders on the basis of previous studies and their associations with the outcomes of a change in effect estimate of more than $10 \%$.

\section{Results}

From June 2017 to March 2018, after deleting rejections and duplicate data, 4176 pregnant women in the first trimester were recruited. Then, deleting the missing data (Loss of follow-up due to miscarriage, transfer, withdrawal, etc.), 2875 pregnant women completed the Pittsburgh Sleep Quality Index both at $12-16$ and $32-36$ gestational weeks, and 2443 completed all the questionnaires, who were included finally (Figure 1).

Total PSQI scores for different gestational weeks (GW) and the increment for participant characteristics are summarized in Table 1. The majority (77.2\%) of pregnant women were between 26 and 35 years of age, and $43.5 \%$ of them possessed of a bachelor's degree or higher. The proportion of overweight and obesity before pregnancy was $16.4 \%$, and $62.8 \%$ of the participants were nulliparous. Regarding the PSQI at 12-16 gestational weeks, bivariate analyses showed that stress, parity, underlying diseases, and a history of abortion were significantly associated with maternal sleep quality, while at $32-36$ gestational weeks, the analyses showed that stress and educational level were significantly associated with maternal sleep quality. For the $\triangle \mathrm{PSQI}$, none of the maternal characteristics was significantly related to sleep quality. In addition, compared with the demographic characteristics of participants that did not complete assessments at both visits, participants in the final included's age of 20-25, high school or lower, overweight or obese and employed were significantly lower, while bachelor's degree or higher and underweight, unemployed groups had a relatively higher proportion.

Table 2 shows that the mean PSQI scores increased from $6.30 \pm 3.01$ in early pregnancy to $7.23 \pm 3.47$ in later pregnancy $(r=0.410, p<0.001)$. Except for the components of "use of sleeping medication" and "daytime dysfunction", five components showed 32-36 gestational weeks 


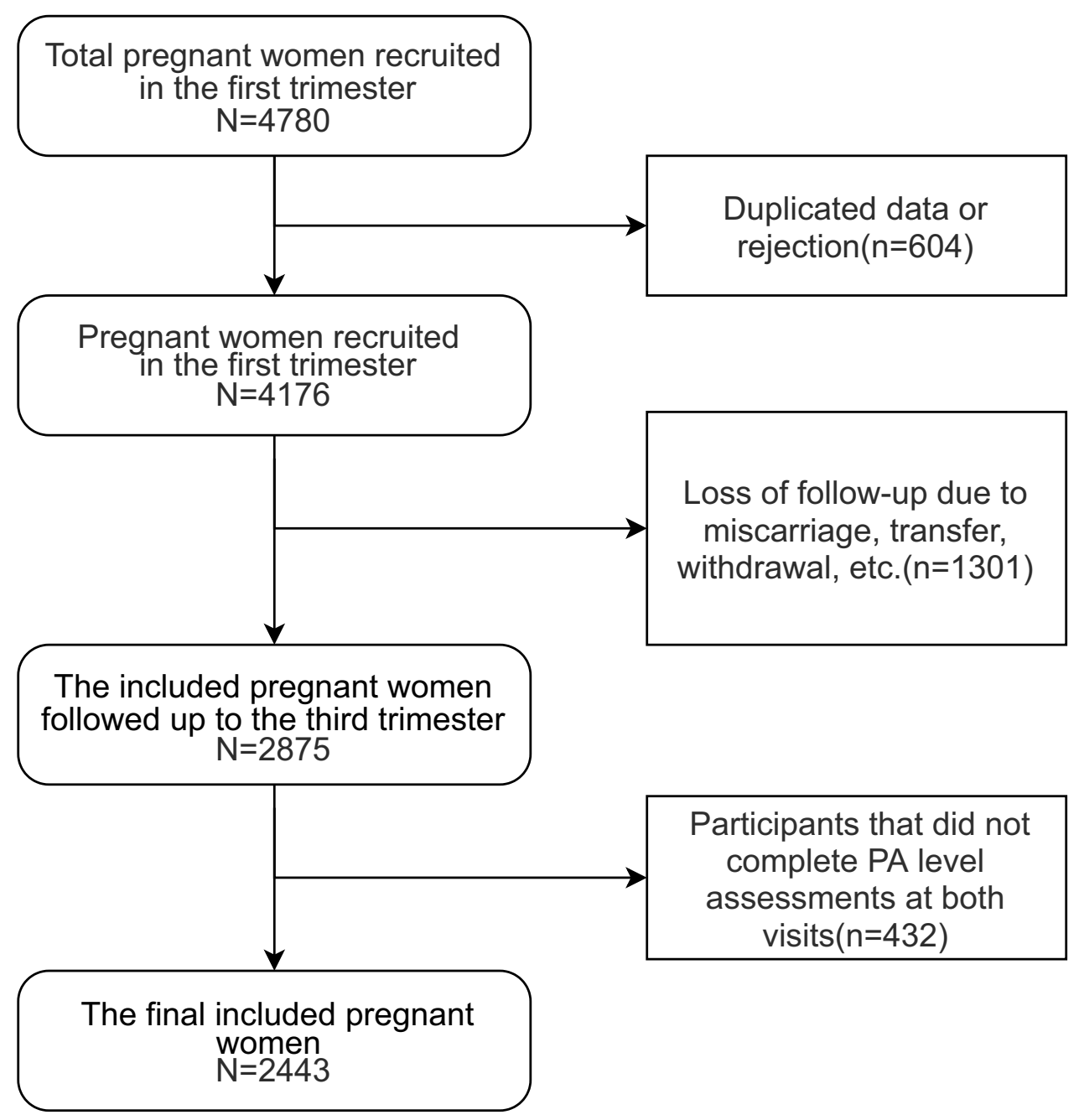

Figure I Flowchart of the selection process for the final included pregnant women.

had higher scores than 12-16 gestational weeks, as did the total PSQI score (all $\mathrm{p}$ values $<0.001$ ). However, the component of daytime dysfunction decreased from 1.26 \pm 0.97 to $1.04 \pm 1.00(\mathrm{p}<0.001)$. The correlation coefficients between PSQI scores at the two pregnancy stages were between 0.238 and 0.410 . At $12-16$ weeks of gestation, $56.7 \%$ of pregnant women received a total PSQI score above 5 points, with the dimensions of subjective sleep quality, sleep disturbance, and daytime dysfunction achieving average scores above 1 . With progressing gestation, approximately $64.4 \%$ of pregnant women had 5 or more points for their total PSQI scores at 32-36 weeks.

PSQI scores in different PA levels at 12-16 gestational weeks had significantly differences $(6.50 \pm 3.02,6.07 \pm 3.00$, $6.47 \pm 2.92$, respectively, $\mathrm{P}<0.01$ ), so have scores at $32-36$ gestational weeks $(7.44 \pm 3.51,7.14 \pm 3.43,6.65 \pm 3.36$, respectively, $\mathrm{P}<0.05)$. The pregnant women who did not increase their PA level had a significantly greater increment in their
PSQI score $(1.08 \pm 3.53, \mathrm{p}=0.001)$ compared to those who had increased PA level $(0.53 \pm 3.52)$. Further comparisons of different PA levels in pairs were conducted using the Bonferroni test. As shown in Figure 2, at 12-16 gestational weeks, only the total PSQI scores for sleep quality were significantly different between the group with a low-level PA and that with a moderate PA level $(p=0.002)$. At $32-36$ gestational weeks, the low PA level group had a significantly higher total PSQI score relative to the other two groups ( $p=0.047$ and 0.043 , respectively). A high PA level in the third trimester significantly reduced the PSQI compared to the group with a low level of PA.

In Table 3, after controlling the covariate factors, compared with low PA level, moderate levels of PA at both 12-16 gestational weeks and 32-36 gestational weeks were significantly associated with lower PSQI scores (AOR= $-0.42,95 \% \quad$ CI:-0.68,-0.16); AOR $=-0.32,95 \%$ CI:-0.63,0.01 , respectively. At $32-36$ weeks, high PA level also 
Table I Total PSQI Scores in Different Gestational Weeks (GW) and Incremented by Participant Characteristics (N=2443)

\begin{tabular}{|c|c|c|c|c|c|c|c|}
\hline \multirow[t]{2}{*}{ Variable } & \multirow[t]{2}{*}{ N (\%) } & \multicolumn{2}{|c|}{ PSQI (I2-16 GW) } & \multicolumn{2}{|c|}{ PSQI (32-36 GW) } & \multicolumn{2}{|l|}{$\triangle P S Q I$} \\
\hline & & Mean士SD & p-value & Mean士SD & p-value & Mean士SD & p-value \\
\hline $\begin{array}{l}\text { Age } \\
\qquad \begin{array}{l}20-25 \\
26-35 \\
\geq 36\end{array}\end{array}$ & $\begin{array}{l}281(11.6) \\
1878(77.2) \\
273(11.2)\end{array}$ & $\begin{array}{l}6.30 \pm 2.98 \\
6.26 \pm 3.14 \\
6.31 \pm 3.01\end{array}$ & $0.68 I$ & $\begin{array}{l}7.22 \pm 3.42 \\
7.43 \pm 3.63 \\
7.24 \pm 3.48\end{array}$ & $0.64 I$ & $\begin{array}{l}0.92 \pm 3.47 \\
1.16 \pm 3.76 \\
0.93 \pm 3.54\end{array}$ & 0.414 \\
\hline $\begin{array}{l}\text { Educational level } \\
\text { High school or lower } \\
\text { Some college } \\
\text { Bachelor's degree or higher }\end{array}$ & $\begin{array}{l}541(22.2) \\
834(34.3) \\
1057(43.5)\end{array}$ & $\begin{array}{l}6.25 \pm 2.98 \\
6.47 \pm 3.11 \\
6.21 \pm 2.96\end{array}$ & 0.159 & $\begin{array}{l}7.24 \pm 3.48 \\
7.50 \pm 3.50 \\
7.03 \pm 3.42\end{array}$ & $0.014^{*}$ & $\begin{array}{l}0.99 \pm 3.81 \\
1.04 \pm 3.60 \\
0.82 \pm 3.34\end{array}$ & 0.398 \\
\hline $\begin{array}{l}\text { Pre-pregnancy BMI }(\mathrm{kg} / \mathrm{m} 2) \\
\text { Underweight }(<18.5) \\
\text { Normal weight }(18.5-23.9) \\
\text { Overweight or obese }(\geq 24)\end{array}$ & $\begin{array}{l}414(17.2) \\
1601(66.4) \\
396(16.4)\end{array}$ & $\begin{array}{l}6.14 \pm 2.88 \\
6.32 \pm 3.05 \\
6.46 \pm 2.96\end{array}$ & 0.320 & $\begin{array}{l}7.10 \pm 3.39 \\
7.22 \pm 3.49 \\
7.49 \pm 3.48\end{array}$ & 0.242 & $\begin{array}{l}0.96 \pm 3.45 \\
0.90 \pm 3.54 \\
1.04 \pm 3.63\end{array}$ & 0.760 \\
\hline $\begin{array}{l}\text { Parity } \\
\text { Primipara } \\
\text { Multipara }\end{array}$ & $\begin{array}{l}1535(62.8) \\
908(37.2)\end{array}$ & $\begin{array}{l}6.16 \pm 3.03 \\
6.52 \pm 2.97\end{array}$ & $0.004 * *$ & $\begin{array}{l}7.16 \pm 3.48 \\
7.35 \pm 3.43\end{array}$ & 0.172 & $\begin{array}{l}1.00 \pm 3.56 \\
0.83 \pm 3.50\end{array}$ & 0.266 \\
\hline $\begin{array}{l}\text { History of abortion } \\
\text { Yes } \\
\text { No }\end{array}$ & $\begin{array}{l}735(30.1) \\
1708(69.9)\end{array}$ & $\begin{array}{l}6.48 \pm 3.00 \\
6.22 \pm 3.01\end{array}$ & $0.042^{*}$ & $\begin{array}{l}7.35 \pm 3.44 \\
7.19 \pm 3.47\end{array}$ & 0.282 & $\begin{array}{l}0.86 \pm 3.48 \\
0.97 \pm 3.57\end{array}$ & 0.500 \\
\hline $\begin{array}{l}\text { Underlying diseases } \\
\text { Yes } \\
\text { No }\end{array}$ & $\begin{array}{l}516(21.1) \\
1927(78.9)\end{array}$ & $\begin{array}{l}6.55 \pm 3.01 \\
6.23 \pm 3.01\end{array}$ & $0.031^{*}$ & $\begin{array}{l}7.39 \pm 3.53 \\
7.19 \pm 3.49\end{array}$ & 0.224 & $\begin{array}{l}0.85 \pm 3.55 \\
0.96 \pm 3.54\end{array}$ & 0.519 \\
\hline $\begin{array}{l}\text { Stress at early stages } \\
\text { Normal } \\
\text { More stressful }\end{array}$ & $\begin{array}{l}2116(86.6) \\
327(13.4)\end{array}$ & $\begin{array}{l}6.13 \pm 2.95 \\
7.39 \pm 3.22\end{array}$ & $<0.001^{* *}$ & $\begin{array}{l}7.09 \pm 3.43 \\
8.18 \pm 3.56\end{array}$ & $<0.001^{* *}$ & $\begin{array}{l}0.96 \pm 3.53 \\
0.80 \pm 3.62\end{array}$ & 0.449 \\
\hline $\begin{array}{l}\text { Working status } \\
\text { Employed } \\
\text { Unemployed }\end{array}$ & $\begin{array}{l}|58|(65.6) \\
828(34.4)\end{array}$ & $\begin{array}{l}6.23 \pm 3.01 \\
6.42 \pm 3.03\end{array}$ & 0.153 & $\begin{array}{l}7.22 \pm 3.43 \\
7.23 \pm 3.52\end{array}$ & 0.984 & $\begin{array}{l}0.99 \pm 3.51 \\
0.81 \pm 3.60\end{array}$ & 0.230 \\
\hline
\end{tabular}

Notes: P-values were calculated using Student's t-tests or one-way ANOVAs, ${ }^{*} \mathrm{p}<0.05,{ }^{* *} \mathrm{p}<0.01$.

significantly decreased PSQI score, with a greater decline than moderate PA levels (AOR=-0.87,95\% CI:-1.57,-0.18). Moreover, with pregnancy progressing from $12-16$ to 32-36 weeks, the increment in PA created a significant decline in PSQI scores of 0.54. We also compared the variable scores subscales of PSQI with increasing PA level under different gestational weeks, the result showed that moderate PA level had the greatest impact on the components of "daytime dysfunction", significantly reducing the score of this dimension. In 32-36 gestational weeks, moderate and high PA levels significantly reduced the scores of the components of "daytime dysfunction" and "Sleep duration", respectively. No significant differences in the subscales' scores of PSQI were found in PA increment.

\section{Discussion}

In the present study, we found that maternal sleep became generally worse as pregnancy progressed, and a relationship existed between the level of pregnant women's physical activity and their sleep quality. At 12-16 weeks of gestation, we observed that moderate PA level was associated with better sleep quality, while at 32-36 weeks of gestation, moderate and high levels of PA would improve sleep quality. Compared with pregnant women who maintained or reduced their PA levels in the third trimester, those who increased their PA levels significantly improved their sleep quality. The corresponding PA level for different gestational weeks might, therefore, be a worthwhile adjunct treatment to combating poor sleep quality during pregnancy. 
Table 2 Comparison of Subjective Sleep Quality Between Pregnant Women in Different Gestational Weeks (N=2875)

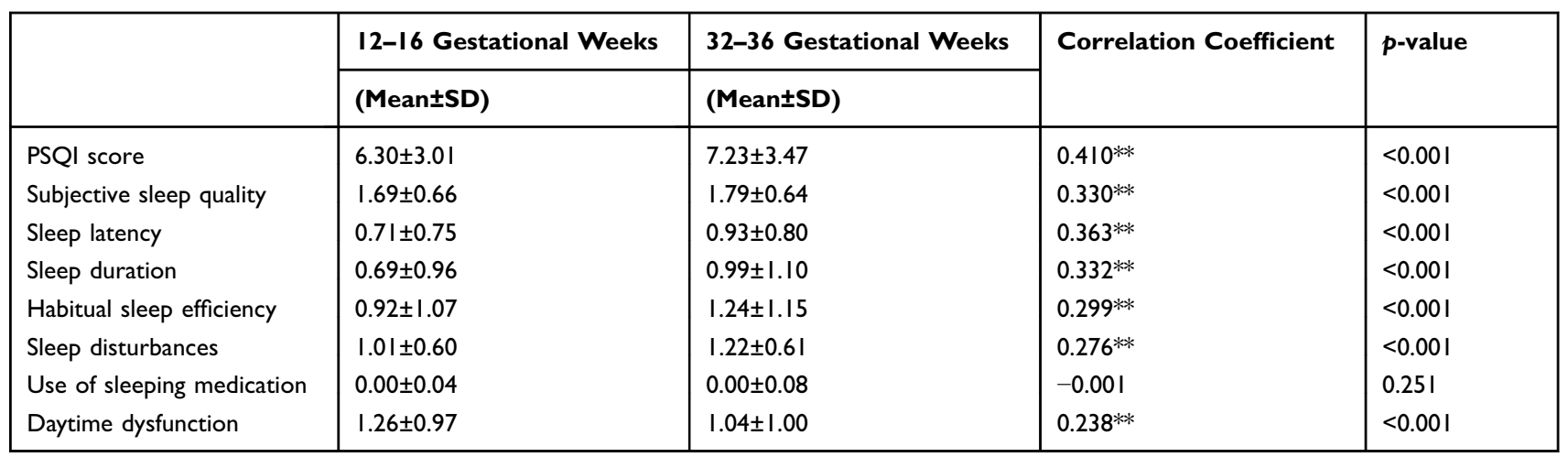

Notes: Comparisons were performed using a paired $t$-test; ${ }^{*}<<0.05$, **p $<0.01$.

That sleep quality in pregnant women was worse in later pregnancy corroborates the results by previous researchers. ${ }^{37,38}$ A meta-analysis indicated ${ }^{38}$ that the component of daytime dysfunction decreased as gestation progressed. There may also be an increase in sleep disturbances in the third trimester, consistent with Sedov's review. ${ }^{39}$ Risk factors for poor maternal sleep quality are complex. In the first trimester, pregnant women may have the symptoms of nocturia, nausea, and vomiting, ${ }^{10}$ and during the third trimester, fetal movements, low back pain, and nocturnal cramps become the principal reasons for poor sleep quality. ${ }^{11}$ Our study showed that a high level of stress, multiparity, underlying diseases, and a history of abortion were associated with poor sleep quality in early pregnancy, and that a high level of stress and moderate educational level were risk factors for poor sleep quality in later gestation. In addition, psychological (eg, stress, depression, and mood disturbance) and behavioral (eg, sleep position) factors have also been reported in prior studies to affect sleep quality. ${ }^{38,40,41}$ These researchers revealed some mechanisms underlying poor sleep quality but most risk factors are difficult to modify, thus, PA levels require further investigation as a factor that can be adjusted by intervention.

The reason we choose the PA level other than intensity in measurement is that PA level combined the intensity and time and it is more flexible for pregnant women to choose the type or prolong the time of their PA activity which are suitable for their physical condition. Our primary aim was to evaluate the association between PA levels or increased levels of PA and sleep quality across pregnancy. The worldwide prevalence of PA during pregnancy is reportedly low, with the main reasons for reduced levels of PA during pregnancy being physical discomfort, complications associated with pregnancy, growth of the woman's body, and
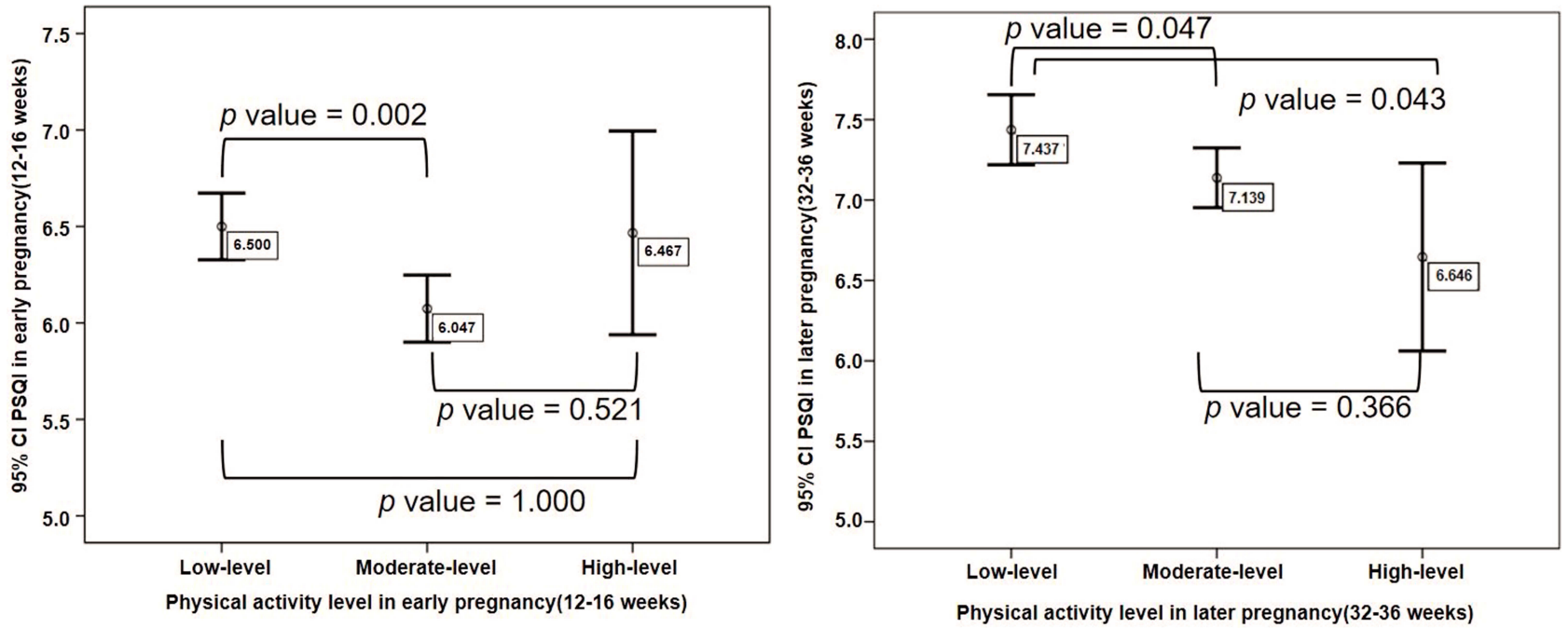

Figure 2 The comparison of PSQI scores between 2 groups of different PA levels at different gestational weeks $(N=2443)$. 


\begin{tabular}{|c|c|c|c|}
\hline هُ & 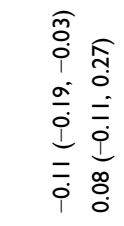 & 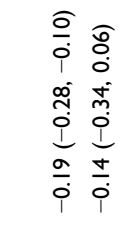 & $\begin{array}{l}0 \\
0 \\
0 \\
0 \\
0 \\
0 \\
1 \\
\overline{0} \\
0\end{array}$ \\
\hline 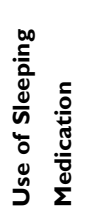 & $\begin{array}{ll}\widehat{\delta} & \overline{0} \\
0 & 0 \\
\circ & - \\
0 & 0 \\
0 & 0 \\
1 & 1 \\
8 & 8 \\
0 & 0\end{array}$ & 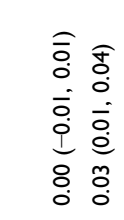 & $\begin{array}{l}\widehat{\overline{0}} \\
\dot{0} \\
\dot{0} \\
0 \\
1 \\
\dot{0} \\
\dot{0}\end{array}$ \\
\hline 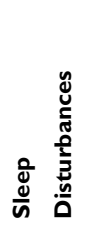 & 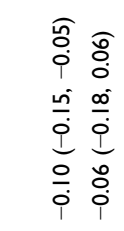 & 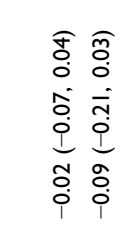 & 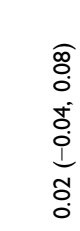 \\
\hline 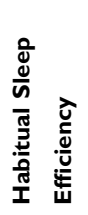 & 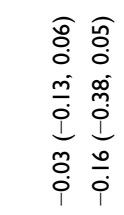 & 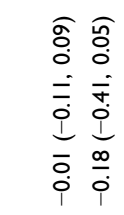 & $\begin{array}{l}0 \\
0 \\
0 \\
0 \\
0 \\
0 \\
0 \\
0 \\
0 \\
0\end{array}$ \\
\hline 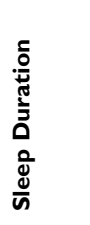 & 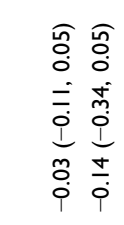 & 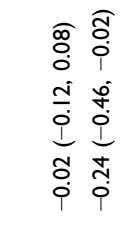 & $\begin{array}{l}\widehat{\sigma} \\
0 \\
0 \\
0 \\
0 \\
0 \\
1 \\
0 \\
0\end{array}$ \\
\hline 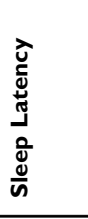 & $\begin{array}{ll}\widehat{\tilde{O}} & \widehat{\bar{T}} \\
0 & 0 \\
= & 0 \\
\overline{0} & 0 \\
1 & 0 \\
0 & 1 \\
0 & 0 \\
0 & 0\end{array}$ & 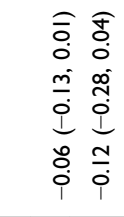 & $\begin{array}{l}\text { 음 } \\
0 \\
0 \\
0 \\
0 \\
1 \\
\text { I } \\
0 \\
0\end{array}$ \\
\hline 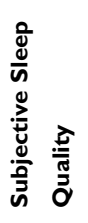 & 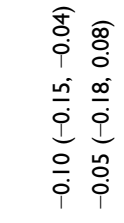 & 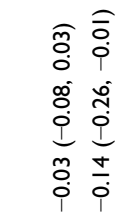 & 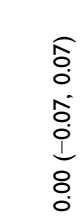 \\
\hline 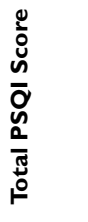 & 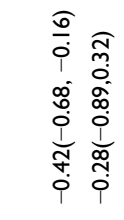 & 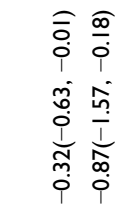 & 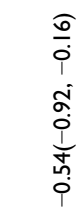 \\
\hline बذ & 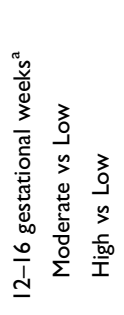 & 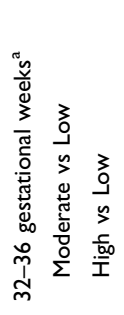 & 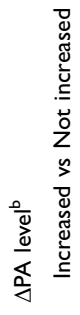 \\
\hline
\end{tabular}

a sense of insecurity. ${ }^{42,43}$ In our cohort study where we measured all parameters twice, we found that lower PA was associated with poorer sleep quality at the same gestational stage. However, moderate PA level in early pregnancy showed positive relation with sleep quality compared with low or high PA level. For high PA level with bad sleep quality, we speculated that the spontaneous abortion rate during early pregnancy is about $3.6 \%-16.0 \%$, which is increased gradually. ${ }^{44-46}$ Therefore, traditional Chinese customs does not recommend high PA level in the first trimester, which may also increase pregnancy response (morning sickness) and result in poor sleep quality. For low PA level with poor sleep quality, as we all know, physical activity and exercise are associated with better sleep quality and fewer sleep disturbances among healthy adults. $^{47-49}$ Considering the health benefits of good sleep quality, pregnant women are an important target group to improve and maybe the low PA level did not improve the sleep quality as much as the moderate PA level. Specifically, pregnant women who increased their levels of PA in later pregnancy showed improved sleep quality, compared with women who maintained or decreased their PA levels in later pregnancy. In addition to improving sleep quality and considering the rapid increase in fetal size and weight, accompanied by the increasing relaxation of maternal ligaments (primarily around the pelvis, but encompassing the entire body), scientifically recommended PA levels at later stages of gestation are closely related to pregnancy health and outcomes. In fact, the total cesarean section rate decreases with the increase in average physical activity in later pregnancy. ${ }^{50,51}$ Pre-eclamptic pregnant women who reduced their exercise levels during the second and third trimesters appeared to have even caused untoward effects to their lower limb muscles, blood pressure, and blood lipids. $^{33}$ To our knowledge, this is the first report entailing prospective assessments performed twice for both sleep quality and PA across pregnancy. These data provide new evidence in support of our hypothesis that adequate PA is a preventive measure against poor maternal sleep quality. The data also augment the small but growing literature regarding PA and pregnant women. Except for the benefits to maternal sleep quality, PA during gestation can additionally be used to prevent and treat gestational and pre-existing diabetes during pregnancy ${ }^{52}$ and used in a positive way for better spontaneous labor. ${ }^{53}$ In fact, moderate-intensity and household/caregiving PA reduced the odds of unplanned cesarean deliveries $^{54}$ in Hispanic women. 
The Chinese Nutrition Society's Dietary Guidelines for Women in Pregnancy (2016) recommends ${ }^{55}$ that healthy pregnant women perform moderate-intensity physical activity for no less than 30 minutes per day. According to the recommendations of the American College of Obstetricians and Gynecologists (ACOG), women with uncomplicated pregnancies should be encouraged to engage in physical activities: moderate-intensity and safe exercise (eg, walking, swimming, stationary cycling, lowimpact aerobics, and strength training) for at least 20-30 minutes per day is recommended. However, the current guidelines for activities during pregnancy in various countries lack the PA-level guidance for women at specific weeks of gestation. Pregnant women in later pregnancy have a single PA mode and show insufficient PA levels, with walking being the main PA mode. ${ }^{56,57}$ The current daily walking recommendations are 5000-7499 steps for low-intensity PA, 10,000-12,499 for moderate-intensity $\mathrm{PA}$, and $>12,500$ steps for high-intensity PA. ${ }^{32}$ Pregnant women can achieve better sleep quality when reaching a moderate level of PA-which combined with the definitions of the IPAQ and the recommendations of the ACOG - equal to 26 minutes of slow walking per day during early pregnancy. Surely, they can choose other physical activity like swimming or yoga for pregnancy or other safe exercise under the supervision to achieve ideal PA level for better sleep quality. Our data, first, provided a novel means to assess the association between groups performing different levels of PA. In addition, to attain better maternal sleep quality in later pregnancy, an appropriately increased level of PA is needed. As gestational weeks progress, the cardiovascular system of pregnant women undergoes adaptive changes, such as increased heart rate and increased blood volume, and it is not advisable to conduct high-intensity PA. However, PA level can be enriched by increasing PA mode and time. We recommended increasing the level of PA slightly on the basis of early pregnancy and carrying out various forms of an appropriate PA mode while walking. Borodulin's study showed that occupational and indoor household physical activity modes and the recreational/indoor household activity cluster were weakly related to sleep duration. ${ }^{58}$ Another study showed ${ }^{59}$ household or caregiving activities were associated with worse sleep quality and shorter sleep duration. High occupational activity and high levels of light-intensity physical activity were associated with lower odds of very poor sleep quality and long sleep duration, respectively. Two of the above studies explored the detailed modes of PA and used different questionnaires to evaluate it, which could be the cause of the difference. It also enlightens us to explore the association between detailed PA and sleep quality among Chinese pregnant women later on. The PA level should be based on the principle of individualization and gradual progress, and the PA level during pregnancy should not exceed the prepregnancy level if there was a routine PA level prior to pregnancy. Several randomized controlled trials ${ }^{60,61}$ of specific exercises for maternal sleep quality have been conducted, and although the sample sizes were small, their results should prove the effectiveness of an appropriate PA level for achieving adequate sleep quality during pregnancy.

Because pregnancy is a dynamic and regular process, it may not be appropriate to use a single module for pregnant women in different gestational weeks. The ACOG emphasizes $^{25}$ the effects of exercise on pregnancyspecific conditions and recommends that outcomes will be studied further, ie, that detailed exercise programs may need to be explored for pregnant women who are at different gestational weeks. Furthermore, in our study, we found that different PA levels were needed at different gestational weeks to ensure better sleep quality. As to certain pregnancy complications such as hypertension or gestational diabetes, research on suitable physical activity levels for different weeks of gestation should also be further conducted.

In the present study, we used longitudinal data and adjusted related confounding factors to explore the changes in sleep quality during pregnancy and the influence of physical activity, and demonstrated that these methods made sleep quality more reliable. Although we found a new relationship between PA and maternal sleep quality within a continuous process, there were still some limitations. We used IPAQ to measure PA levels during pregnancy, which was brief and did not assess specific physical activity types and energy expenditure. Although IPAQ has been applied in pregnant women, we categorized physical activity by using thresholds defined in nonpregnant populations, which is the major limitation. In addition, administering the survey for PA and sleep quality at a second time might not represent the entire dynamic process of pregnancy. Thus, multiple and specific surveys should be explored in further studies. Additionally, since we did not obtain data on PA level or sleep quality through objective measurements, we instead used self-report questionnaire, which might add recall bias to the results. The 
final included pregnant women have relatively higher educational level and underweight, which limits the generalizability of the results. Therefore, future studies can be conducted in large sample size based on the results measured by objective instruments (like actigraphy).

\section{Conclusion}

Our study revealed worse sleep quality in later gestational weeks. The moderate PA level had the potential for improvement of sleep quality both in the first and third trimester and high PA level was also beneficial to improve sleep quality of pregnant women in later pregnancy. Increases in PA levels from early pregnancy to later pregnancy can prospectively predict better changes in sleep quality. We recommend moderate PA level in early pregnancy and to increase their PA level in late pregnancy to improve sleep quality.

\section{Author Contributions}

Huijing Shi and Qing Yang, the two corresponding authors contributed equally to this work. Huijing Shi and Liwei Tan made a major contribution to the conception and design of this study. Huijing Shi and Qing Yang mainly applied for grant funding and organized the data collection. Liwei Tan and Jiaojiao Zou were mainly responsible for the data analysis, wrote the draft and revised it critically for important intellectual content. Huijing Shi and Yunhui Zhang made a substantial contribution to the manuscript's improvement. All authors made contributions to conception and design, acquisition of data, or analysis and interpretation of data; took part in drafting the article or revising it critically for important intellectual content; gave final approval of the version to be published; and agree to be accountable for all aspects of the work.

\section{Funding}

This study was supported by Municipal Education Commission-Gaofeng Public Health Grant Support and the Fourth Round of the Three-Year Public Health Action Plan of Shanghai (15GWZK0402).

\section{Disclosure}

The authors report no conflicts of interest in this work.

\section{References}

1. Okun ML, Christine Dunkel S, Glynn LM. Poor sleep quality is associated with preterm birth. Sleep. 2011;34(11):1493. doi:10.5665/ sleep. 1384
2. Naghi I, Keypour F, Ahari SB, et al. Sleep disturbance in late pregnancy and type and duration of labour. J Obstet Gynaecol. 2011;31(6):489-491. doi:10.3109/01443615.2011.579196

3. Iranpour S, Kheirabadi GR, Esmaillzadeh A, et al. Association between sleep quality and postpartum depression. J Res Med Sci. 2016;21(1):110. doi:10.4103/1735-1995.193500

4. Duntley PSP, Macones GA. Sleep deprivation during pregnancy and maternal and fetal outcomes: is there a relationship?. Sleep Med Rev. 2010;14(2):107-114. doi:10.1016/j.smrv.2009.05.001

5. Gay CL, Richoux SE, Beebe KR, et al. Sleep disruption and duration in late pregnancy is associated with excess gestational weight gain among overweight and obese women. Birth. 2017;44(2):173. doi:10.1111/birt.12277

6. Wang W, Zhong C, Zhang Y, et al. Shorter sleep duration in early pregnancy is associated with birth length: a prospective cohort study in Wuhan, China. Sleep Med. 2017;34:99-104. doi:10.1016/j. sleep.2017.03.013

7. Jomeen J, Martin CR. Assessment and relationship of sleep quality to depression in early pregnancy. J Reprod Infant Psychol. 2007;25 (1):87-99. doi:10.1080/02646830601117308

8. Warland J, Dorrian J, Morrison JL, et al. Maternal sleep during pregnancy and poor fetal outcomes: a scoping review of the literature with meta-analysis. Sleep Med Rev. 2018;41:S1087079217300138. doi:10.1016/j.smrv.2018.03.004

9. Leigh T, Sarah-Jane P, Bronwyn S, et al. Prevalence of abnormal sleep duration and excessive daytime sleepiness in pregnancy and the role of socio-demographic factors: comparing pregnant women with women in the general population. Sleep Med. 2014;15(12):1477-1483. doi:10.1016/ j.sleep.2014.07.007

10. Reichner CA. Insomnia and sleep deficiency in pregnancy. Obstet Med. 2015;8(4):168-171. doi:10.1177/1753495X15600572

11. Ruiz JR, Perales M, Pelaez M, et al. Supervised exercise-based intervention to prevent excessive gestational weight gain: a randomized controlled trial. Mayo Clin Proc. 2013;88(12):1388-1397. doi:10.10 16/j.mayocp.2013.07.020

12. Yang Y, Mao J, Ye Z, et al. Determinants of sleep quality among pregnant women in China: a cross-sectional survey. Matern Fetal Neonatal Med. 2017;31(22):1. doi:10.1080/14767058.2017.1359831

13. Ruiz-Robledillo N, Canário C, Dias CC, et al. Sleep during the third trimester of pregnancy: the role of depression and anxiety. Psychol Health Med. 2015;20(8):927-932. doi:10.1080/13548506.2015.1017508

14. Tsai SY, Lin JW, Wu WW, et al. Sleep disturbances and symptoms of depression and daytime sleepiness in pregnant women. Birth. 2016;43(2):176. doi:10.1111/birt.12215

15. Wołyńczyk-Gmaj D, Różańska-Walędziak A, Ziemka S, et al. Insomnia in pregnancy is associated with depressive symptoms and eating at night. J Clin Sleep Med. 2017;13(10). doi:10.5664/jcsm.6764

16. Rodriguez-Blanque R, Sánchez-García JC, Sánchez-López AM, et al. The influence of physical activity in water on sleep quality in pregnant women: a randomised trial. Women Birth. 2017;31(1): S1871519216302670. doi:10.1016/j.wombi.2017.06.018

17. Rayward AT, Burton NW, Brown WJ, et al. Associations between changes in activity and sleep quality and duration over two years. Med Sci Sports Exerc. 2018;50(12):2425-2432. doi:10.1249/ MSS.0000000000001715

18. Irish LA, Kline CE, Rothenberger SD, et al. A 24-hour approach to the study of health behaviors: temporal relationships between waking health behaviors and sleep. Ann Behav Med. 2014;47(2):189-197. doi:10.1007/s12160-013-9533-3

19. Chee W, Kim S, Tsai HM, et al. Decreasing sleep-related symptoms through increasing physical activity among Asian American midlife women. Menopause. 2018;26(2):1. doi:10.1097/GME.000000000000 1178

20. Banno M, Harada Y, Taniguchi M, et al. Exercise can improve sleep quality: a systematic review and meta-analysis. Peerj. 2018;6(7): e5172. doi: $10.7717 /$ peerj.5172 
21. Spörndly-Nees S, Lindberg E, Lindberg E. High or increasing levels of physical activity protect women from future insomnia. Sleep Med 2017;32:22. doi:10.1016/j.sleep.2016.03.017

22. Baker JH, Rothenberger SD, Kline CE, et al. Exercise during early pregnancy is associated with greater sleep continuity. Behav Sleep Med. 2016:1. doi:10.1080/15402002.2016.1228649.

23. Avcı F, Avcı F, Kardaş S. Factors affecting general sleep pattern and quality of sleep in pregnant women. Turk J Obstet Gynecol. 2015;12 (1):1-5. doi:10.4274/tjod.22120

24. Piercy KL, Troiano RP, Ballard RM, et al. The physical activity guidelines for Americans. JAMA. 2018;320(19):2020. doi:10.1001/ jama.2018.14854

25. Activity P, Pregnancy ED. Committee opinion no. 650: physical activity and exercise during pregnancy and the postpartum period. Obstet Gynecol. 2015;126(6):e135. doi:10.1097/AOG.0000000000001214

26. Skouteris H, Wertheim EH, Germano C, Paxton SJ, Milgrom J. Assessing sleep during pregnancy: a study across two time points examining the pittsburgh sleep quality index and associations with depressive symptoms. Women's Health Issues. 2009;19(1):0-51. doi:10.1016/j.whi.2008.10.004

27. Buysse DJ, Reynolds CF, Monk TH, et al. The Pittsburgh sleep quality index: a new instrument for psychiatric practice and research. Psychiatry Res. 1989;28(2):193-213. doi:10.1016/0165-1781(89)90047-4

28. Group QIPAI. International physical activity questionnaire [EB/OL]. [Dec 2020]. Available from: https://www.academia.edu/5346814/ Guidelines_for_Data_Processing_and_Analysis_of_the International_Physical_Activity_Questionnaire_IPAQ_Short_and Long_Forms_Contents.

29. Helou K, El Helou N, Mahfouz M, et al. Validity and reliability of an adapted arabic version of the long international physical activity questionnaire. BMC Public Health. 2018;18(1):49. doi:10.1186/ s12889-017-4599-7

30. Craig CL, Marshall AL, Bauman AE, et al. International physical activity questionnaire: 12-country reliability and validity. Med Sci Sports Exerc. 2003;35(8):1381-1395. doi:10.1249/01.mss.0000078924.61453.fb

31. Sanda B, Vistad I, Haakstad L, et al. Reliability and concurrent validity of the international physical activity questionnaire short form among pregnant women. BMC Sports Sci Med Rehabil. 2017;9(1). doi:10.1186/s13102-017-0070-4

32. Catrine T-L, Yoshiro H, Robert PP, et al. Revisiting "how many steps are enough?". Med Sci Sports Exerc. 2008;(Supplement). doi:10.1249/ mss.0b013e31817c7133

33. Wang X. The Research of Relationship Between Nourishment and Movement with Preeclampsia [Master]. Shang Dong University; 2008.

34. Zhou BF, Meta-Analysis C. Predictive values of body mass index and waist circumference to risk factors of related diseases in Chinese adult population. Chin J Epidemiol. 2002.

35. Zhang Z. Behavioral Medicine Scale Manual. Chinese Medicine Multimedia Press; 2005.

36. Gao Y, Yang YF, Hong Q, et al. Development of life events scale for pregnant women and analysis of its reliability and validity. Chin J Women Child Health Res. 2010;6(21):743-746.

37. Tomfohr LM, Buliga E, Letourneau NL, et al. Trajectories of sleep quality and associations with mood during the perinatal period. Sleep. 2015;38(8):1237-1245. doi:10.5665/sleep.4900

38. Sedov ID, Cameron EE, Madigan S, et al. Sleep quality during pregnancy: a meta-analysis. Sleep Med Rev. 2018;38:168-176. doi:10.1016/j.smrv.2017.06.005

39. Ladyman C, Signal TL. Sleep health in pregnancy: a scoping review. Sleep Med Clin. 2018;13(3):307-333. doi:10.1016/j.jsmc.2018.04.004

40. Skouteris H, Germano C, Wertheim EH, et al. Sleep quality and depression during pregnancy: a prospective study. J Sleep Res. 2010;17(2):217-220. doi:10.1111/j.1365-2869.2008.00655.x

41. Mindell JA, Cook RA, Nikolovski J. Sleep patterns and sleep disturbances across pregnancy. Sleep Med. 2015;16(4):483-488. doi:10.1016/j.sleep.2014.12.006
42. Pivarnik J, Chambliss H, Clapp J, et al. Impact of physical activity during pregnancy and postpartum on chronic disease risk. Med Sci Sports Exerc. 2006;38(5):989-1006. doi:10.1249/01.mss.0000218147.51025.8a

43. Hegaard HK, Kjaergaard H, Damm PP, et al. Experiences of physical activity during pregnancy in Danish nulliparous women with a physically active life before pregnancy. A qualitative study. $B M C$ Pregnancy Childbirth. 2010;10(1):1-10. doi:10.1186/1471-2393-10-33

44. Zhou A, Liu XH, Department of Gynecology. A study on the correlation between the early intrauterine pregnancy outcome and serum HCG \& P levels. Chin Med Pharm. 2015

45. Xu JPWH, Guo CL. Analysis of influencing factors and evaluation of pregnancy outcome of threatened abortion. Chin Remedies Clinics. 2015;15(9):1317-1320.

46. Wang FHHH, Tan YY. Observation of the curative effect treatment of dydrogesterone and progesterone and human chorionic gonadotropin treatment of threatened abortion. China J Pharm Econ. 2015;10 (6):45-47.

47. Kronholm E, Härmä M, Hublin C, et al. Self-reported sleep duration in Finnish general population. J Sleep Res. 2006;15(3):276-290. doi:10.1111/j.1365-2869.2006.00543.x

48. Youngstedt SD. Effects of exercise on sleep. Clin Sports Med. 2005;24(2):355-365. doi:10.1016/j.csm.2004.12.003

49. Atkinson G, Davenne D. Relationships between sleep, physical activity and human health. Physiol Behav. 2007;90(2-3):0-235. doi:10.1016/j.physbeh.2006.09.015

50. Hegaard HK, Pedersen BK, Birgitte Bruun N, et al. Leisure time physical activity during pregnancy and impact on gestational diabetes mellitus, pre-eclampsia, preterm delivery and birth weight: a review. Acta Obstet Gynecol Scand. 2011;86(11):1290-1296. doi:10.1080/ 00016340701647341

51. Poston L, Caleyachetty R, Cnattingius S, et al. Preconceptional and maternal obesity: epidemiology and health consequences. Lancet Diabetes Endocrinol. 2016;4(12):1025. doi:10.1016/S2213-8587(16) 30217-0

52. Di Biase NBS, Balducci S, Lencioni C, et al. Review of general suggestions on physical activity to prevent and treat gestational and pre-existing diabetes during pregnancy and in postpartum. Nutr Metab Cardiovasc Dis. 2019;29(2):115-126. doi:10.1016/j.numecd.2018.10.013

53. Ussher M, Lewis S, Aveyard P, et al. Physical activity for smoking cessation in pregnancy: randomised controlled trial. BMJ. 2014;350 (may14 16):h2145. doi:10.1136/bmj.h2145

54. Russo LM, Harvey MW, Pekow P, et al. Physical activity and risk of cesarean delivery in hispanic women. $J$ Phys Act Health. 2019;16 (2):116-24. doi:10.1123/jpah.2018-0072

55. Group CNSDGRECWaCsDGREW. Dietary guidelines for pregnant women (2016). Chin J Perinat Med. 2016;19(9):641-648.

56. Investigation LY. Analysis of current situation of women's physical activity in later pregnancy in Cheng Du. Matern Child Health Care China. 2015;30(32):5648-5650.

57. Ren Y. Investigation on the exercise behavior of pregnant women during pregnancy and pre-pregnancy. General Care. 2018;16(34):119-120.

58. Katja BKM, Kelly RE, Monda K, et al. Physical activity and sleep among pregnant women. Paediatr Perinat Epidemiol. 2010;24 (1):45-52. doi:10.1111/j.1365-3016.2009.01081.x

59. Hawkins M, Marcus B, Pekow P, et al. Physical activity and sleep quality and duration during pregnancy among hispanic women: estudio PARTO. Behav Sleep Med. 2018:1-14. doi:10.1080/15402002.2018.1518225.

60. Kocsis I, Szilágyi T, Turos J, et al. Effect of a gymnastics program on sleep characteristics in pregnant women. Taiwan J Obstet Gynecol. 2017;56(2):204-209. doi:10.1016/j.tjog.2017.02.001

61. Özkan SA, Rathfisch G. The effect of relaxation exercises on sleep quality in pregnant women in the third trimester: a randomized controlled trial. Complement Ther Clin Pract. 2018;32:79-84. doi:10.1016/j.ctcp.2018.05.008 


\section{Publish your work in this journal}

Nature and Science of Sleep is an international, peer-reviewed, open access journal covering all aspects of sleep science and sleep medicine, including the neurophysiology and functions of sleep, the genetics of sleep, sleep and society, biological rhythms, dreaming, sleep disorders and therapy, and strategies to optimize healthy sleep.
The manuscript management system is completely online and includes a very quick and fair peer-review system, which is all easy to use. Visit http://www.dovepress.com/testimonials.php to read real quotes from published authors. 